\title{
A Review of In Vitro Drug Release Test Methods for Nano-Sized Dosage Forms
}

\author{
Susan D'Souza \\ Sunovion Pharmaceuticals Inc, Marlborough, MA 01752, USA \\ Correspondence should be addressed to Susan D’Souza; dr_ssdsouza@yahoo.com
}

Received 16 June 2014; Revised 15 August 2014; Accepted 15 August 2014; Published 20 November 2014

Academic Editor: Cornelia M. Keck

Copyright (C) 2014 Susan D’Souza. This is an open access article distributed under the Creative Commons Attribution License, which permits unrestricted use, distribution, and reproduction in any medium, provided the original work is properly cited.

\begin{abstract}
This review summarizes the methods used to study real-time $\left(37^{\circ} \mathrm{C}\right)$ drug release from nanoparticulate drug delivery systems and establish an IVIVC. Since no compendial standards exist, drug release is currently assessed using a variety of methods including sample and separate (SS), continuous flow (CF), dialysis membrane (DM) methods, and a combination thereof, as well as novel techniques like voltametry and turbidimetry. This review describes the principle of each method along with their advantages and disadvantages, including challenges with set-up and sampling. The SS method allows direct measurement of drug release with simple set-up requirements, but sampling is cumbersome. With the CF method, sampling is straightforward but the set-up is time consuming. Set-up as well as sampling is easier with the DM, but it may not be suitable for drugs that bind to the membrane. Novel methods offer the possibility of real-time drug release measurement but may be restricted to certain types of drugs. Of these methods, Level A IVIVCs have been obtained with dialysis, alone or in combination with the sample and separate technique. Future efforts should focus on developing mathematical models that describe drug release mechanisms as well as facilitate formulation development of nano-sized dosage forms.
\end{abstract}

\section{Introduction}

Ever since reports documenting the utility of polycyanoacrylate and poly- $\varepsilon$-caprolactone nanocapsules for ocular administration were published over two decades ago, several publications have highlighted the benefits of using nanosized dosage forms for medical and imaging purposes [13]. Indeed, advantages such as improved drug solubility and stability, enhanced performance as well as increased efficacy have been well established with nanoparticulate preparations [4]. The increasing interest in nanotechnology based drug delivery systems has been a key factor in the design and development of numerous novel dosage forms and complex delivery therapies such as liposomes, nanoemulsions, nanocrystals, polymeric nanoparticles, solid lipid nanoparticles, nanofibers, and dendrimers, to treat a variety of disease states [5-7]. As an example, researchers have investigated nanoparticles of Fenofibrate for the treatment of hypercholesterolemia and Cyclosporine nanoparticles against cancer [8, 9]. Unsurprisingly, several nanoparticulate preparations are currently undergoing clinical investigation for the delivery of a wide range of therapeutics like antibiotics, antigens, cytostatics, and so forth, via the intramuscular, subcutaneous, oral, and intravenous route [10, 11]. A few examples of currently marketed nanotechnology based dosage forms are listed in Table 1.

By the ISO definition, the size for these formulations can range between 1 and $100 \mathrm{~nm}$. Since sizes below $10 \mathrm{~nm}$ have a greater propensity for renal clearance and tissue extravasations, and larger sizes are quickly opsonized via the macrophages of the reticuloendothelial system, the 10$100 \mathrm{~nm}$ size range is considered optimal for nanoparticulate preparations [12]. Hence, for medical and therapeutic purposes, a range of less than $10-100 \mathrm{~nm}$ (in at least one dimension) for nanoparticulates appears to be generally accepted, with a few exceptions where sizes greater than $100 \mathrm{~nm}$ may be applicable $[4,13]$. Due to their small size, nano-sized dosage forms possess an unusually large surface-to-volume ratio that alters the chemical, physical, and biological properties of the dosage form allowing them to cross cell and tissue 
TABLE 1: List of a few currently marketed nanoparticulate products.

\begin{tabular}{|c|c|c|}
\hline Brand Name & API & Indication \\
\hline DaunoXome & Daunorubicin & Kaposi’s sarcoma \\
\hline Depocyt & Cytarabine & Lymphomatous meningitis \\
\hline Myocet & Doxorubicin & Breast cancer \\
\hline Oncaspar & $\begin{array}{l}\text { PEG- } \\
\text { asparaginase }\end{array}$ & $\begin{array}{l}\text { Acute Lymphoblastic } \\
\text { Leukemia }\end{array}$ \\
\hline Restasis & Cyclosporine & Chronic dry eye disease \\
\hline Triglide & Fenofibrate & Hypercholesterolemia \\
\hline Emend & Aprepitant & $\begin{array}{l}\text { Chemotherapy induced } \\
\text { nausea and vomiting }\end{array}$ \\
\hline
\end{tabular}

barriers, thereby altering the pharmacokinetics and pharmacodynamics of the therapeutic agent. This unique aspect of nanoparticulate preparations has been exploited to deliver therapeutics to specific cells, organs, and other challenging in vivo targets. Another consequence of enhanced delivery to the target site is an increase in potency of the drug, including the potential for elevated toxicity due to the carrier material, possibly leading to reduced safety. Therefore, determination of product quality and performance becomes a crucial aspect during nanoparticulate dosage form development.

As with most dosage forms, product quality and performance may be verified through several in vivo and/or in vitro experiments [14]. Of these, drug release kinetics provides critical information about dosage form behavior and is a key parameter used to assess product safety and efficacy. Due to the expense, time, labor, and need for human subjects/animals when performing in vitro measurements of drug release kinetics, in vitro release is gaining greater attention as a surrogate test for product performance. Indeed, in vitro release testing is commonly used as a predictor of in vivo behavior, historically with traditional dosage forms like capsules and tablets (i.e., dissolution), and more recently with novel dosage forms like injectable biodegradable microspheres and implants [15-17]. Generally, in vitro release studies are performed at $37^{\circ} \mathrm{C}$ (physiological temperature), though in some instances testing at elevated temperatures has been explored to characterize drug release from a variety of dosage forms $[18,19]$. Some of the key objectives of in vitro release testing are one or more of the following:

(a) assessing the effect of formulation factors and manufacturing methods on the drug product,

(b) routine assessment of quality control to support batch release,

(c) substantiating product label claims,

(d) establishing an in vitro in vivo correlation/relationship (IVIVC/R),

(e) assuring product sameness under the SUPAC guidelines,

(f) as a compendial requirement $[20,21]$.
Without exception, in vitro release testing is an important analytical tool that is used to investigate and establish product behavior during the various stages of drug product development, as well as life cycle management. When designed appropriately, an in vitro release profile can reveal fundamental information on the dosage form and its behavior, as well as provide details on the release mechanism and kinetics, enabling a rational and scientific approach to drug product development. Understandably, for complex dosage forms like nanoparticulates, in vitro release testing assumes greater significance.

Despite the great strides in design and development of nano-sized dosage forms, no compendial or regulatory standards exist for in vitro release testing. Although there have been attempts to use the existing USP apparatus for in vitro drug assessment of nanoparticles, the set-ups were designed primarily for oral and transdermal products and as such pose many challenges during a release study. Hence, several in vitro release methods, both compendial and noncompendial, have been utilized and reported. Certainly, the area of in vitro testing for nanoparticulates lags behind the advances realized in drug product development.

Given the urgent need for improved product safety while maintaining quality of novel dosage forms like nanoparticles, the past decade witnessed a series of international in vitro release workshops co-sponsored by the AAPS (American Association of Pharmaceutical Scientists), US FDA (Food and Drug Administration), FIP (Federation International Pharmaceutique), and several other scientific groups and agencies [22-25]. The outcomes of these workshops were published as position papers with the general consensus that dissolution or in vitro release testing was an important tool in pharmaceutical development and quality control for a plethora of dosage forms, both traditional and novel. Further, the attendees highlighted the fact when compared to traditional formulations, the characteristics of novel/special dosage forms including site and mode of administration was vastly different, and, hence, appropriate consideration should be taken during selection of the apparatus, release medium, agitation (flow rate), and temperature. In summary, the workshops concluded that, along with a few other novel dosage forms, nanoparticulate preparations were categorized as those "dosage forms requiring more work before a (dissolution) method can be recommended" [22, 23].

In a separate endeavor, the Nanotechnology Risk Assessment Working Group in the Center for Drug Evaluation and Research (CDER) within the US FDA, published a regulatory note outlining the risk assessment and management process on a hypothetical nanomaterial drug, to be administered orally [4]. In this example, the Working Group identified "dissolution/release rate" as a risk factor that may impact the evaluation of quality, safety, and efficacy of the drug product containing nano-sized drug, that is, during ingestion and in vitro release/dissolution. Hence, the utility of in vitro release testing, from a drug product development as well as regulatory standpoint, cannot be ignored.

Thus, the purpose of this review is to provide the reader with a summary of existing in vitro release methods for nano-sized dosage forms along with their strengths and 
shortcomings. It is hoped that this review will serve as a guideline for developing suitable techniques for assessment of exploratory formulations, clinical and commercial dosage forms, and establishment of an in vitro in vivo correlation (IVIVC).

\section{In Vitro Release Methods}

Much attention has been devoted to the development of suitable apparatus to assess in vitro release from nanoparticulates. However, additional considerations, like release media selection, agitation, and so forth, cannot be overlooked. In contrast to oral dosage forms where release media typically mimics $\mathrm{pH}$ of the gastrointestinal tract, selection of release media for nano-sized dosage forms will vary depending on the site of administration as well as the site of action of the formulation, and thus simulation of in vivo conditions may be difficult. In general, selection of release media for nanoparticulate preparation is generally based on drug solubility and stability, assay sensitivity, and the method used. Though maintenance of sink conditions is preferable, nonsink conditions have been employed. Agitation, frequently utilized to prevent aggregation of dosage forms during an in vitro release study, will depend on the apparatus used. Similarly, sampling and buffer replacement (total or partial) techniques are also based on the type of in vitro method used.

As such, drug release from nano-sized dosage forms can be assessed using one of the following three categories, namely, sample and separate (SS), continuous flow (CF), and dialysis membrane (DM) methods. More recently, apparatus that combine the principles of either the SS and DM or CF and DM have also been reported. Lastly, a few novel methods that use voltametry, turbidimetry, and so forth are discussed. For each of these methods, a brief description is provided along with adaptations, additional considerations, advantages, and disadvantages.

2.1. Sample and Separate. In the SS method, the nanoparticulate dosage form is introduced into the release media that is maintained at a constant temperature, after which drug release is assessed by sampling of the release media (filtrate or supernatant) or the nanoparticles. From literature, there are several adaptations to the SS method, with differences noted in set-up, container size, mode of agitation, and sampling techniques.

Commonly reported set-ups include USP I (basket), USP II (paddle), or vials and generally depend on the volume of release media used in the in vitro release study [7, 38-40]. For instance, vials were used when the volume of release media was small (1-15 mL) and in vitro release vessels were employed with larger volumes $(600-900 \mathrm{~mL})[5,7,38,40,41]$.

In addition to determining the type of set-up, container size also influences the mode of agitation used in an in vitro release study with the SS method. With small sized dosage forms like nanoparticles, agitation of the release media is critical to the in vitro release process as it reduces the potential for aggregation and enhances wetting, thereby reducing the impact of these factors on the in vitro release rate [38]. While agitation of the release media is easily accomplished via the USP I or USP II apparatus, media contents in vials have been agitated using alternate techniques. For example, Danhier et al. and Li et al. used magnetic stirrers with Paclitaxel and BSA nanoparticles, respectively, while Prabha et al. reported the use of orbital shakers with DNA nanoparticles [42-44].

Drug release is monitored by physically separating the nanoparticles from the release media, followed by analysis of the former or the latter [7, 41]. While several authors have documented the use of syringe filters to achieve physical separation between the release media and nanoparticles, the small size of nanoparticles has necessitated the use of high energy separation techniques like centrifugation, ultracentrifugation, and ultrafiltration [40, 42, 45-47]. For filtration, syringe filters with pore size as large as $0.45 \mu \mathrm{m}$ have been used for withdrawing supernatant to monitor drug release of small molecules such as Celecoxib [40]. In comparison, high energy separation techniques have been reported with larger biomolecules like Insulin, BSA (Bovine Serum Albumin), and DNA [43, 44, 48]. Once separated, drug release is generally monitored by sampling a part of the supernatant, or decanting the entire supernatant content at periodic intervals $[7,49]$. In other instances, separation was followed by analysis of the nanoparticles (destructive technique) [41]. After sampling, an equal amount of fresh release media or buffer is added to the set-up so that sink conditions are maintained for the duration of the in vitro release study.

In general, the SS method provides a direct approach to monitor drug release. With this method, most sample setups, agitation modes, and sampling techniques are reasonably straightforward and simple. However, due to the small size of nanoparticulate dosage forms, several practical challenges have been noted. For instance, aggregation of nanoparticles during in vitro release appears to be a key concern. Additionally, while sampling techniques like filtration seem fairly reasonable in principle, clogging of filters during sampling, adsorption of drug to the filter, and so forth, have been reported [50]. Indeed, difficulty in physical separation even with high energy techniques, continued drug release during the high energy separation process, and so forth, are some of the challenges often observed during sampling [38, 45]. Although sink conditions are recommended with the SS method, nonsink conditions have been reported to be more discriminatory with poorly soluble drugs [51]. Nevertheless, as with microparticulate dosage forms, the SS method offers researchers a simple and straightforward approach to monitor in vitro release from nano-sized dosage forms.

2.2. Continuous Flow. In the CF method, drug release from the nanoparticulate dosage form is monitored using the USP IV apparatus or a modification thereof. Drug release occurs as a result of buffer or media constantly circulating through a column containing the immobilized dosage form and is monitored by collecting the eluent at periodic intervals (Figure 1). 


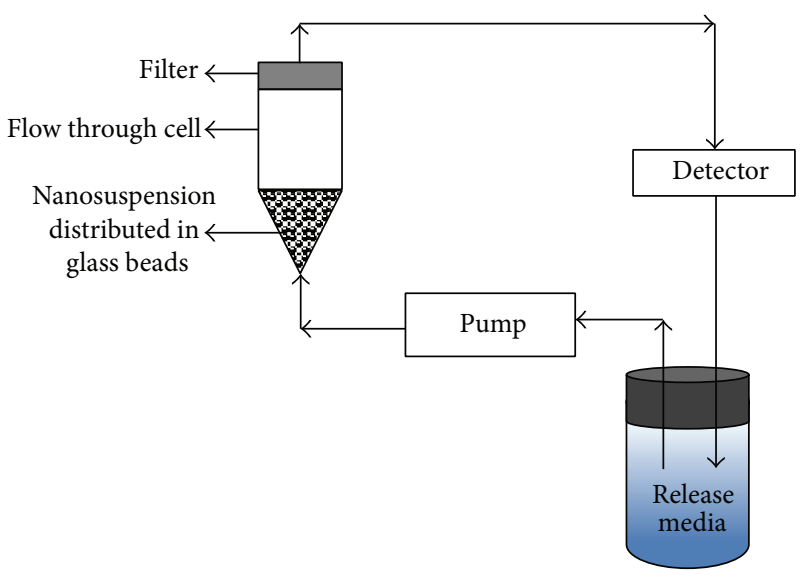

Figure 1: Continuous flow set-up in closed loop configuration.

Unlike the widely used SS method, only a few examples of the CF method have been reported for nano-sized dosage forms $[38,52]$. For example, the release profiles of amorphous nanoparticles and an unprocessed crystalline form of Cefuroxime Axetil, a BCS II cephalosporin antibiotic, were evaluated using the CF method as well as other methods described below [38]:

(a) USP I (basket): $900 \mathrm{~mL}$ buffer at $100 \mathrm{rpm}$;

(b) USP II (paddle): $900 \mathrm{~mL}$ buffer at $100 \mathrm{rpm}$;

(c) USP IV (flow through cell): $900 \mathrm{~mL}$ buffer at a flow rate of $1.6 \mathrm{~mL} / \mathrm{min}$ (peristaltic pump, closed loop) through a cell (internal diameter $=25 \mathrm{~mm}$ ) and $0.2 \mu \mathrm{m}$ membrane disc filter;

(d) dialysis bag (MWCO $12 \mathrm{kDa}$, inner volume $=7 \mathrm{~mL}$ ) placed into a USP II (paddle) in vitro release tester (outer volume $=900 \mathrm{~mL}$, paddle $\mathrm{rpm}=100$ ).

The results revealed that complete drug release was achieved only with the USP II paddle and USP IV apparatus, release profiles with the USP IV method being well separated.

Immobilization of the nanoparticles was thought to be a factor contributing to the discriminatory results obtained with the USP IV apparatus.

Similarly, in an experiment on nano- and micro-particle loaded strip films of another BCS II drug, Griseofulvin, in vitro release was performed using two methods [52]:

(a) USP I (basket): 500 and $900 \mathrm{~mL}$ media at 50, 100, and $150 \mathrm{rpm}$;

(b) USP IV (flow through cell): $100 \mathrm{~mL}$ media at flow rates of 4,8 , and $16 \mathrm{~mL} / \mathrm{min}$ (peristaltic pump, closed loop) through a cell (internal diameter $=22.6 \mathrm{~mm}$ ) and $0.2 \mu \mathrm{m}$ membrane disc filter with the strip film loaded in 6 different configurations.

While complete release was observed only with USP I and configuration B (strip film positioned in the cell on top of round glass beads) of the USP IV method, results with configurations A (strip film positioned in the cone section

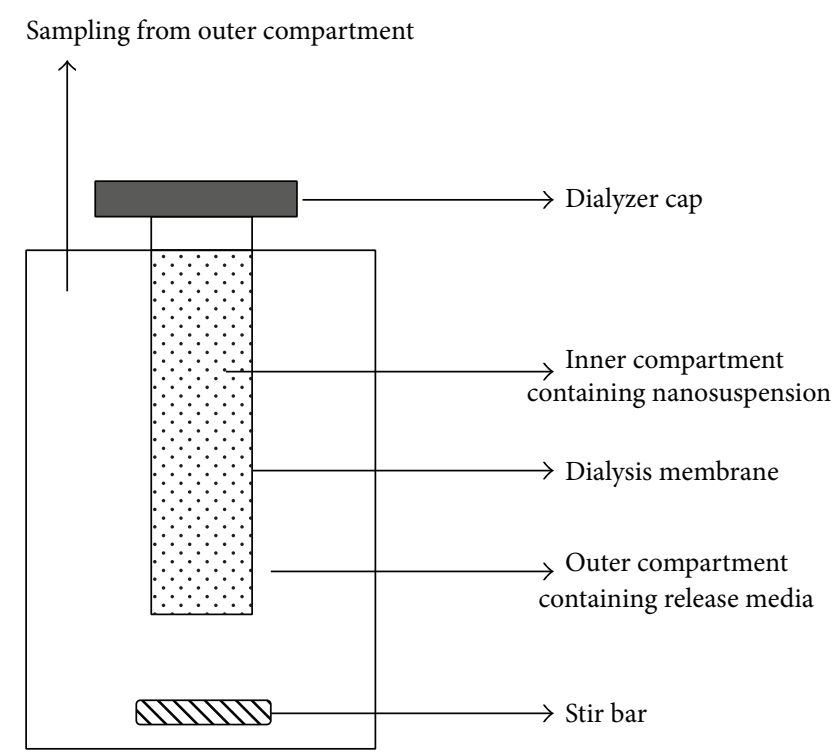

FIgURE 2: "Regular dialysis" set-up (adapted from [21]).

of the cell without round glass beads) and C (strip film sandwiched between round glass beads) of USP IV at the highest flow rate were more discriminatory than all the other release profiles obtained in the in vitro study.

In comparison to nanoparticulate dosage forms where literature on the use of USP IV methodology is sparse, several salient aspects of the CF method can be inferred from its use in microparticulate dosage forms [21]. As an example, flow rates used in the CF method depend on the type of pump (peristaltic versus syringe) as well as the filters used (membrane versus ultrafilters). Low flow rates have been known to be a key factor causing slow or incomplete release from dosage forms [21]. In general, the availability of automated equipment has simplified routine sampling and media replacement with the the CF method in closed (recirculating media) and open (nonrecirculating media) loop systems. However, the CF methods suffer from several disadvantages that include instrument costs, difficulty in setup, filter clogging, adsorption to the filter and glass beads, and difficulty in maintaining a constant flow rate leading to wide variability in results.

2.3. Dialysis Method. Of all the methods used to assess drug release from nano-sized dosage forms, the dialysis method (DM) is the most versatile and popular. In this method, physical separation of the dosage forms is achieved by usage of a dialysis membrane which allows for ease of sampling at periodic intervals. As with the other methods, several adaptations of the DM have been reported in literature with key differences in set-up, container size, and molecular weight cut-off (MWCO).

Of the variety of DM set-ups used, the most commonly cited is the dialysis bag (regular dialysis), other adaptations being the reverse dialysis and side-by-side dialysis set-up $[26,53,54]$ (Figures 2-4). With the regular dialysis technique, 


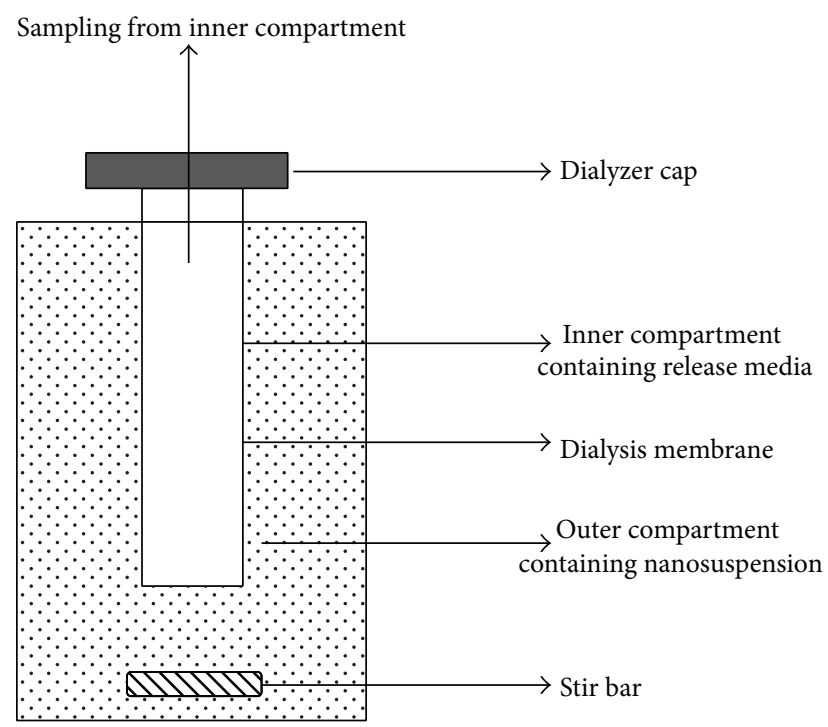

FIGURE 3: "Reverse dialysis" set-up.

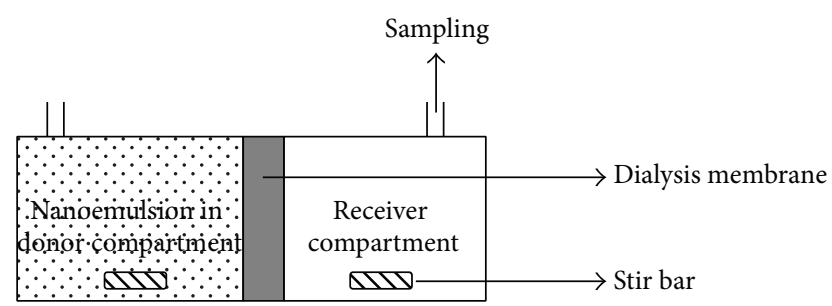

Figure 4: “Side-by-side dialysis" set-up (adapted from [26]).

the nanoparticles are introduced into a dialysis bag containing release media (inner media/compartment) that is subsequently sealed and placed in a larger vessel containing release media (outer media/compartment), agitated to minimize unstirred water layer effects $[32,55]$. In general, the volume enclosed in a dialysis bag (inner media) is significantly smaller than the outer media. For instance, inner media volumes reported in literature range from 1 to $10 \mathrm{~mL}$, whereas the outer media volume is much greater, typically around $40-$ $90 \mathrm{~mL}[32,53,55]$. Thus, container size will depend on the total volume of release media required for the in vitro release study. In the regular dialysis technique, drug released from the nanoparticles diffuses through the dialysis membrane to the outer compartment from where it is sampled for analysis (Figure 2). In contrast, with the reverse dialysis setup, the nanoparticles are placed in the outer compartment (agitated to minimize the unstirred water layer) and the inner compartment is sampled for drug release (Figure 3) $[54,56,57]$. Other adaptations of the DM include the sideby-side dialysis set-up where donor and receiver cells, both containing equal volumes of media agitated with a magnetic stirrer, are separated by a dialysis membrane, and sampling occurs from the receiver cell (Figure 4) and a vertical Franz diffusion cell $[26,58,59]$.
As with other methods, drug solubility in the release media is essential to its transport across the dialysis membrane. Apart from release media, the importance of selecting an appropriate molecular weight cut-off (MWCO) for the dialysis membrane cannot be overlooked. Indeed, the basic premise of the DM is that drug that is released from the dosage form will diffuse rapidly from one compartment, through the membrane, and enter the second compartment from where it is sampled for analysis. Thus, membranes with a sufficiently high membrane MWCO are often selected for in vitro release studies so that drug transport is not a limiting factor. As an example, in a study with 3 dosage forms of Indomethacin (e.g., nanoparticles and nanocapsules using poly-E-caprolactone, and a submicron emulsion), Calvo et al. noted that nearly $85 \%$ of drug was released within 2 hours and complete release was achieved in 4 hours when a $12 \mathrm{kDa}$ membrane was used [54]. However, the rationale for selecting a MWCO is rather subjective. For instance, a MWCO as high as $10-14 \mathrm{kDa}$ was used to study drug release of small molecules like Risperidone and Indomethacin, and a MWCO as low as $1 \mathrm{kDa}$ though suitable for assessing in vitro release of a large molecule, pDNA, proved to be limiting for the diffusion of Cefuroxime Axetil, a cephalosporin antibiotic $[32,38,55,60]$. In general, the MWCO should be sufficiently large to permit drug transport.

The ease of set-up and sampling with the DM make it a very simple and straightforward technique to study drug release from a wide variety of nano-sized dosage forms like nanospheres, liposomes, emulsions, nanosuspensions, and so forth [53-55]. However, issues have been reported with the regular dialysis method. If incorrectly sealed, leakage of media and dosage form may occur from both ends of the dialysis bag set-up. Incomplete release data may be observed if nonsink conditions exist or equilibration times are high [38]. On the other hand, the difference in equilibration times can be exploited as a discriminatory tool to distinguish release behavior between fast and slow releasing dosage forms [50]. Another factor to be considered is that the DM cannot be used with drugs that bind to the dialyzing membrane. As stated in a review paper on microparticulate dosage forms, it is recommended that the suitability of the dialyzing membrane be assessed prior to use [21, 61].

2.4. Combination Methods. A few publications have modified the set-ups used in the SS, CF, and DM methods to evaluate drug release from nano-sized dosage forms. In most of these reports, the set-up of the SS method is combined with a dialyzer to allow ease of sampling. In other publications, the $\mathrm{DM}$ and the CF set-up is used to assess in vitro release from nanoparticulates.

As an example, Mottaleb et al. compared release profiles of Ibuprofen from liposomes and lipid nanocapsules and nanoparticles using the regular dialysis method (dialysis bag) and modified USP I (basket) set-up where the shaft was affixed with a glass basket having a dialysis membrane at the bottom, that is, glass basket dialysis (GBD), and placed in a larger vessel (Figure 5) [27]. Sampling was performed from the outer vessel. Unlike the dialysis bag that had a large 


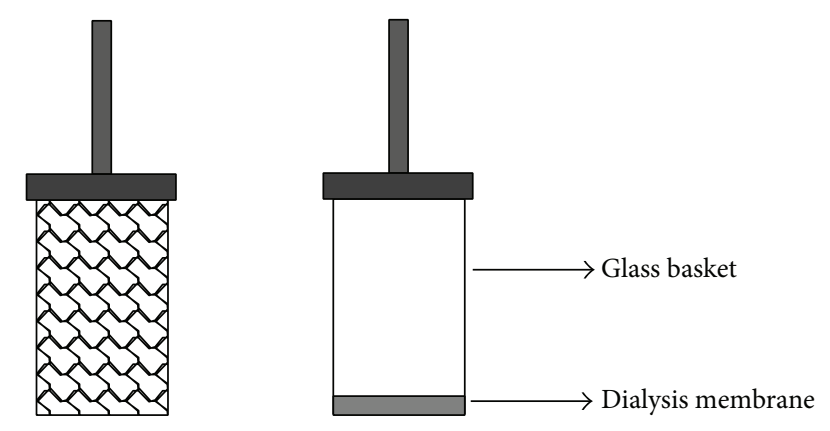

FIGURE 5: Comparison of USP I (left) and glass basket dialysis (right) set-ups (adapted from [27]).

membrane surface area, the GBD was able to discriminate differences between release profiles from different formulations (lipid nanocapsules, nanoparticles, liposomes). In another study, Lobenberg et al. compared drug release from Rifampicin and Moxifloxacin hydrochloride nanoparticles with the regular dialysis method (dialysis bag) as well as an adaptation of USP I (basket), where a cylinder containing a dialysis membrane at the bottom was attached to the basket shaft [62]. Results from this study showed that modified USP I set-up was more discriminatory in nature and could be attributed to the reduced surface area of the dialysis membrane. In yet another modification of the USP I (basket) set-up, a dialysis bag containing nanostructured lipid carriers of Artemether was placed inside the basket and drug release monitored over time [63]. Though most studies have utilized the USP I set-up, others have used the USP II (paddle) set-up in combination with the DM. For example, Cao et al. affixed dialysis bags containing Silybin meglumine nanoparticles onto a USP II (paddle) apparatus and assessed drug release in five different release media $[33,35]$.

A combination of a DM, previously reported by Kostanski et al., and CF method was utilized to assess drug release from 5-Fluorouracil loaded BSA nanoparticles [28, 64]. The setup comprised a glass tube (with a dialysis membrane at the bottom) that was placed into a larger vessel containing buffer (Figure 6). Sampling as well as buffer replacement was performed from the larger vessel with the aid of two pumps having the same flow rate. The combined DM-CF set-up was able to demonstrate sustained levels of 5-Fluorouracil and the absence of burst release from the BSA nanoparticles. An alternate dialysis-CF method utilized a dialysis adaptor inside the CF set-up (Figure 7) [29].

2.5. New Methods. Apart from the commonly reported SS, $\mathrm{CF}$, and DM methods, a few alternate approaches have been utilized to monitor drug release. A majority of these appear to be targeted towards electroactive drugs. While the techniques may differ in principle or application, they possess a few common elements in that physical separation of the dosage form from the release media is not required. Additionally, these techniques offer the possibility of measurement under real-time conditions.

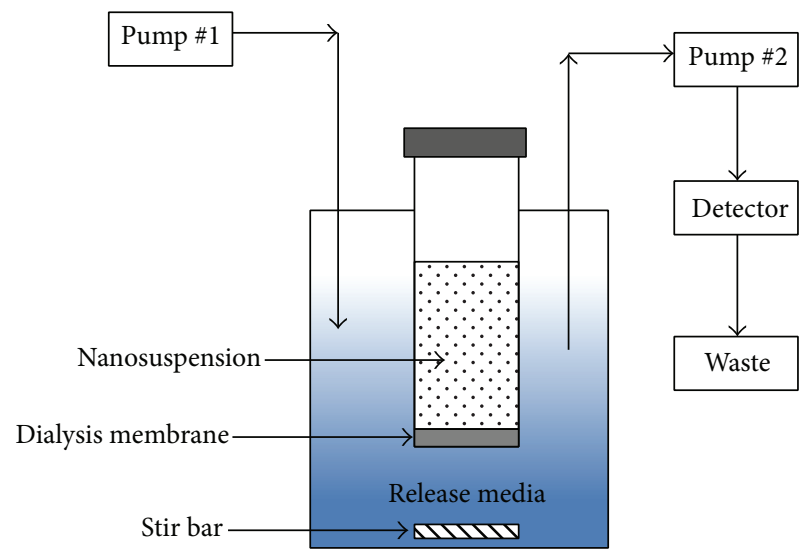

FIGURE 6: Continuous flow dialysis set-up with two pumps (adapted from [28]).

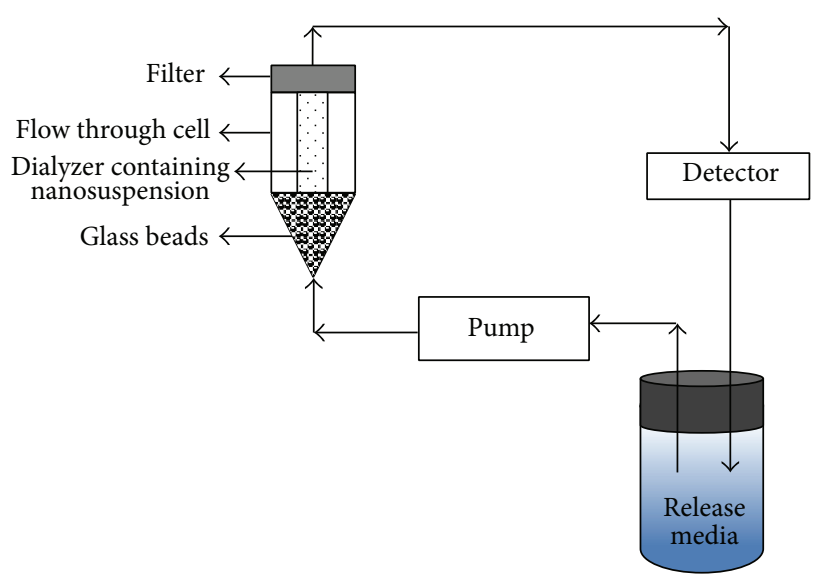

FIgURE 7: Dialysis adapter inside a continuous flow set-up (adapted from [29]).

As such, electrochemical methods offer the possibility of rapid in situ measurements while avoiding the interference caused by the presence of undissolved dosage form in the release media. In one such approach, a potentiometric drug selective electrode was used to monitor real-time release of Procaine hydrochloride from $\mathrm{pH}$-responsive nanogels [65]. In another report by Mora et al., a highly sensitive repetitive square-wave voltammetric technique was utilized to measure the redox reaction of Doxorubicin hydrochloride (Figure 8) [30]. Similarly, differential pulse polarography was used to assess continuous drug release from Piroxicam, Chloramphenicol, and Diazepam [66, 67]. Apart from the fact that only electroactive drugs can be measured by the above methods, these methods suffer from additional drawbacks. For example, the sensitivity as well as responsiveness of each technique varies greatly. Also, the sensors used may be specific to a particular drug.

Nonelectrochemical methods like calorimetry, turbidimetry, and laser diffraction have also been evaluated as in vitro release methods. Solution calorimetry was employed by Kayaert et al. during in vitro release measurement of Naproxen, Cinnarizine, and Compound A (investigational 


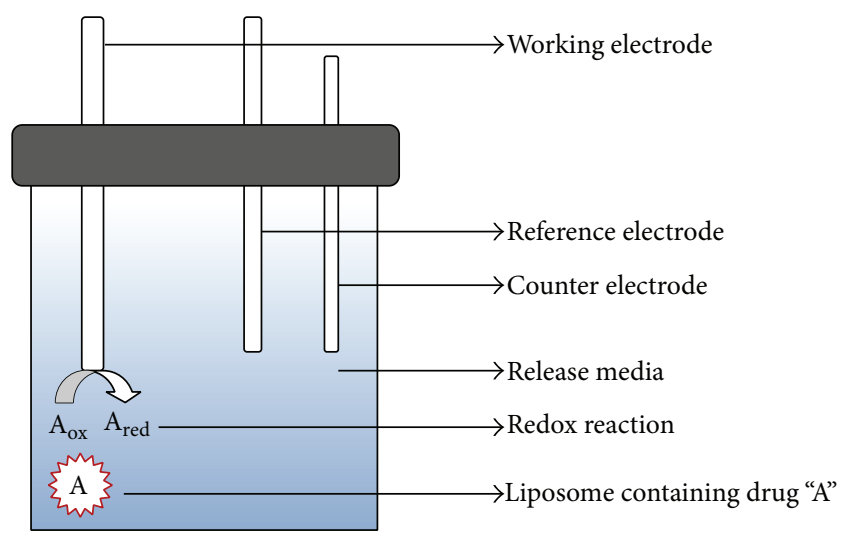

FIGURE 8: Electrochemical cell set-up for real-time measurement of drug release (adapted from [30]).

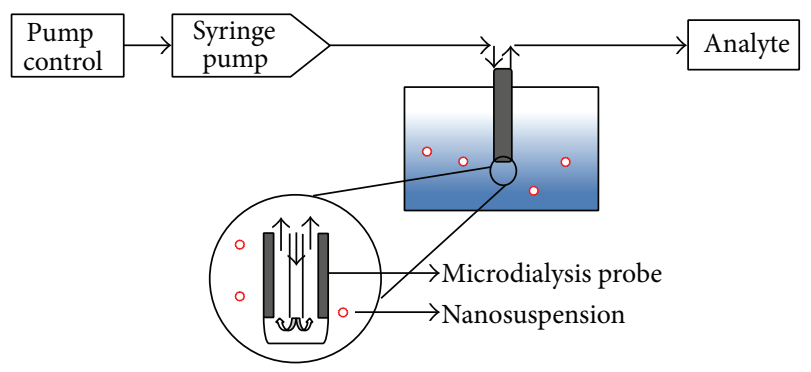

FIgURE 9: Microdialysis set-up (adapted from [31]).

API) from nanocrystals [68]. The principle of this technique is based on detection of the net proportion of heat change during in vitro release. Challenges with calorimetric measurements include long equilibration times. Further, the heat produced by all the processes needs to be considered. Turbidimetric and laser diffraction approaches utilizing the change light scattering properties of nanoparticles dispersed in release media have also been utilized [69, 70]. Concerns with these methods include the long equilibration times, limited range of particle size, and initial concentration of samples that can be used.

Helle et al. designed a novel online system for evaluating the drug release from Indomethacin and Beclomethasone dipropionate nanoparticles. In this method, nanoparticles are packed into small column which is connected to multiport modulation valve attached to an HPLC system allowing for rapid analysis [71]. Other new methods reported in literature include a vertical diffusion Franz cell and an in vitro lipolysis model [37, 72]. Microdialysis, another setup utilized to measure drug release from nanoparticules, is based on passive diffusion of drug through a concentration gradient across a semipermeable membrane (Figure 9) [31, 47]. Drug release can be measured in real-time conditions using analytical methods like HPLC. However, care should be taken when surfactants are used in the release media, as recovery may be affected.

\section{Modeling Drug Release}

Very few publications have attempted to describe drug release profiles from nanoparticulate dosage forms using mathematical models. This is not surprising given that nano-sized dosage forms are complex and drug release evaluations are not straightforward. However, a few authors have discussed the importance of mathematical models and endeavored to characterize drug release. For instance, Barzegar-Jalali et al. suggested the use of reciprocal powered time (RPT) as general model to analyze the complex nature of drug release from nanoparticles, with alternate approaches being the Weibull and log-probability models [73]. In another report, Zeng et al. developed a three-parameter model that considers reversible drug-carrier interaction as well as diffusional drug release from liposomes [74, 75].

Use of mathematical models to describe drug release profiles offers several advantages. It permits the elucidation of drug release mechanisms and can be used to guide formulation development efforts. Further, model parameters can serve to represent as well as compare in vitro release profiles. However, caution must be used when applying mathematical models to complex drug release mechanisms.

\section{IVIVC}

The US FDA defines IVIVC as "A predictive mathematical model describing the relationship between an in vitro property of an extended release dosage form (usually the rate or extent of drug in vitro release or release) and a relevant in vivo response, for example, plasma drug concentration or amount of drug absorbed" [76]. Thus, establishment of an IVIVC, described as a correlation between in vitro release and in vivo behavior, enhances the utility of an in vitro study. From the 1997 FDA guidance document, an IVIVC will reduce the regulatory burden by paring the number of in vivo studies needed for product approval. Additionally, it promotes the setting of clinically relevant in vitro release specifications [76].

The FDA guidance also categorizes IVIVCs into three levels. Level A represents a linear or nonlinear point-topoint relationship between the in vitro and in vivo release profiles. If the relationship is nonlinear, suitable modeling or scaling is required. Of the three IVIVC levels, Level A describes the highest correlation and is most commonly used to obtain a biowaiver. On the other hand, a Level B correlation is a comparison of summary parameters such as the mean in vitro dissolution time with mean in vivo dissolution time or mean residence time (MRT). Since several in vivo curves will produce a similar MRT value or mean in vitro dissolution time, Level B correlations are not as discriminatory as Level A, but acceptable for an IVIVC. A Level C correlation describes a relationship between an in vitro release parameter (e.g., \% dissolved at a particular time) and a pharmacokinetic parameter (e.g., $C_{\max }$ ). However, a Level $C$ correlation is not descriptive of the complete shape of the in vivo release profile and, though acceptable, seldom used.

As such, the FDA guidance is intended for oral extended release products. However, principles of the FDA guidance 
TABLE 2: Examples of IVIVC with nano-sized dosage forms in animal models.

\begin{tabular}{|c|c|c|c|c|}
\hline Drug and dosage form & In vitro release set-up & In vivo studies & IVIVC type & Reference \\
\hline Indomethacin gelatin nanoparticles & Dialysis bags & $\begin{array}{l}\text { Wistar Albino } \\
\text { rats }\end{array}$ & Level A & {$[32]$} \\
\hline $\begin{array}{l}\text { Silybin meglumine hollow-sphere } \\
\text { mesoporous silica nanoparticles }\end{array}$ & Combination (USP I—dialysis bag) & Beagle dogs & Level A & {$[33]$} \\
\hline $\begin{array}{l}\text { Silybin 72-hour SLB and } \\
\text { PSNs (porous silica nanoparticles) }\end{array}$ & Combination (USP I_dialysis bag) & Beagle dogs & Level A & {$[35]$} \\
\hline $\begin{array}{l}\text { Simvastatin nanostructured lipid } \\
\text { carriers and nanoparticles }\end{array}$ & Dialysis bag & Balb/C mice & Level A & {$[34]$} \\
\hline Fenofibrate nanosuspension & USP I apparatus & $\begin{array}{c}\text { Sprague } \\
\text { Dawley rats }\end{array}$ & Level A & {$[36]$} \\
\hline Fenofibrate lipid matrix particles & In vitro lipolysis model & $\begin{array}{c}\text { Sprague } \\
\text { Dawley rats }\end{array}$ & Rank order & {$[37]$} \\
\hline
\end{tabular}

TABLE 3: Level A IVIVC with two Silybin preparations (data from reference [35]).

\begin{tabular}{lcc}
\hline Formulation & $\%$ absorbed equals & $R^{2}$ \\
\hline 72-hour SLB & $1.1379 \times \%$ dissolved +8.8688 & 0.9831 \\
Silybin PSNs (porous silica nanoparticles) & $0.8915 \times \%$ dissolved -10.152 & 0.9831 \\
\hline
\end{tabular}

have been used to demonstrate an IVIVC from several nonoral dosage forms, including nano-sized preparations. Most published literature on IVIVC compares the in vitro release behavior with the in vivo absorption profile, the latter calculated by the FDA recommended Wagner-Nelson method $[76,77]$. Once the fraction absorbed is computed, a correlation is obtained by comparing it with the in vitro release profile and the type of IVIVC (Level A, level B, etc.) is determined.

Over the last decade, a few reports on IVIVC from nanosized dosage forms have been published (Table 2). In some instances, a rank order correlation was observed while, in others, a Level A IVIVC was established with some or all of the formulations evaluated in the study. As an example, a rank order correlation was observed with three preparations of Fenofibrate with drug release and absorption from $100 \mathrm{~nm}$ lipid matrix particles $>$ microparticles $>$ control (crystalline suspension) [37]. In another study on Fenofibrate nanosuspensions, $\mathrm{Xu}$ et al. attempted to elucidate the absorption mechanism using in situ gut perfusion model [36]. Data analysis between in vitro dissolution $(P)$, in situ intestinal absorption $(F)$, and in vivo absorption $\left(F_{a}\right)$ demonstrated a Level A correlation $\left(R^{2}>0.95\right)$. As a result, the authors concluded that the in situ intestinal perfusion model could be a predictor of in vivo pharmacokinetic behavior for the nanosuspension.

In a recent report, Kumar et al. investigated the relationship between in vitro and in vivo release from Indomethacin gelatin nanoparticles administered orally to Wistar albino rats [32]. Study results indicated that a good correlation was obtained by plotting the fraction absorbed in vivo (WagnerNelson method) against the fraction released, the latter performed using regular dialysis (dialysis bags) (Figure 10). The Wagner-Nelson method was also used in another publication

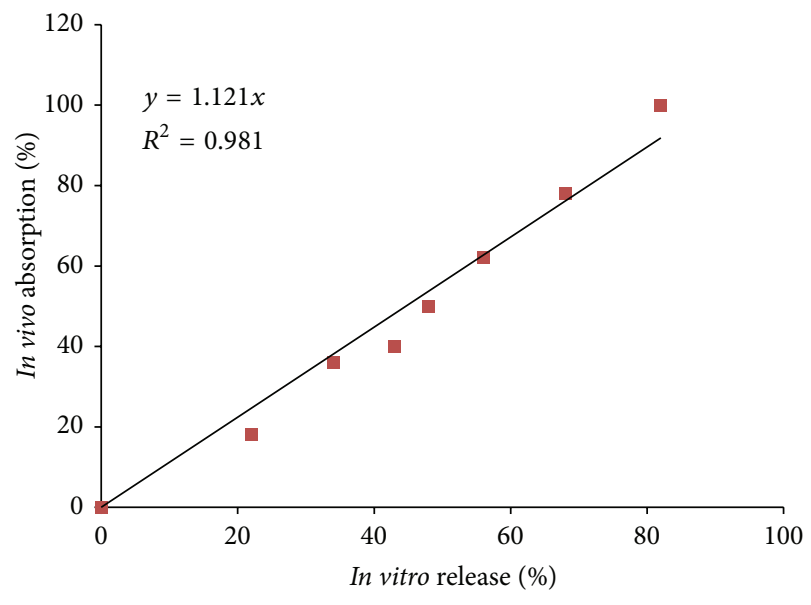

FIGURE 10: Level A IVIVC from Indomethacin gelatin nanoparticles using regular dialysis (redrawn from [32]).

where the in vivo absorption from Silybin meglumine hollowsphere mesoporous silica nanoparticles, dosed orally to beagle dogs, was compared with in vitro results obtained using a combination USP I (paddle) dialysis bag set-up [33]. Of the five different release media used in the study, a Level A IVIVC $\left(R^{2}>0.97\right)$ was obtained with $0.06 \mathrm{M} \mathrm{Na}_{2} \mathrm{CO}_{3}$ and $0.08 \mathrm{M}$ $\mathrm{Na}_{2} \mathrm{CO}_{3}$ solutions (Figure 11). In another study with Silybin, an IVIVC was achieved with a 72-hour release formulation of Silybin (72-hour SLB) that consisted of a solid dispersion, gel matrix, and porous silica nanoparticles (PSNs) (Table 3) [35]. Similar to the earlier study, the authors compared in vivo release from beagle dogs with in vitro release assessed using a combination USP I (paddle) dialysis bag set-up. In another paper, Tiwari et al. demonstrated a Level A 

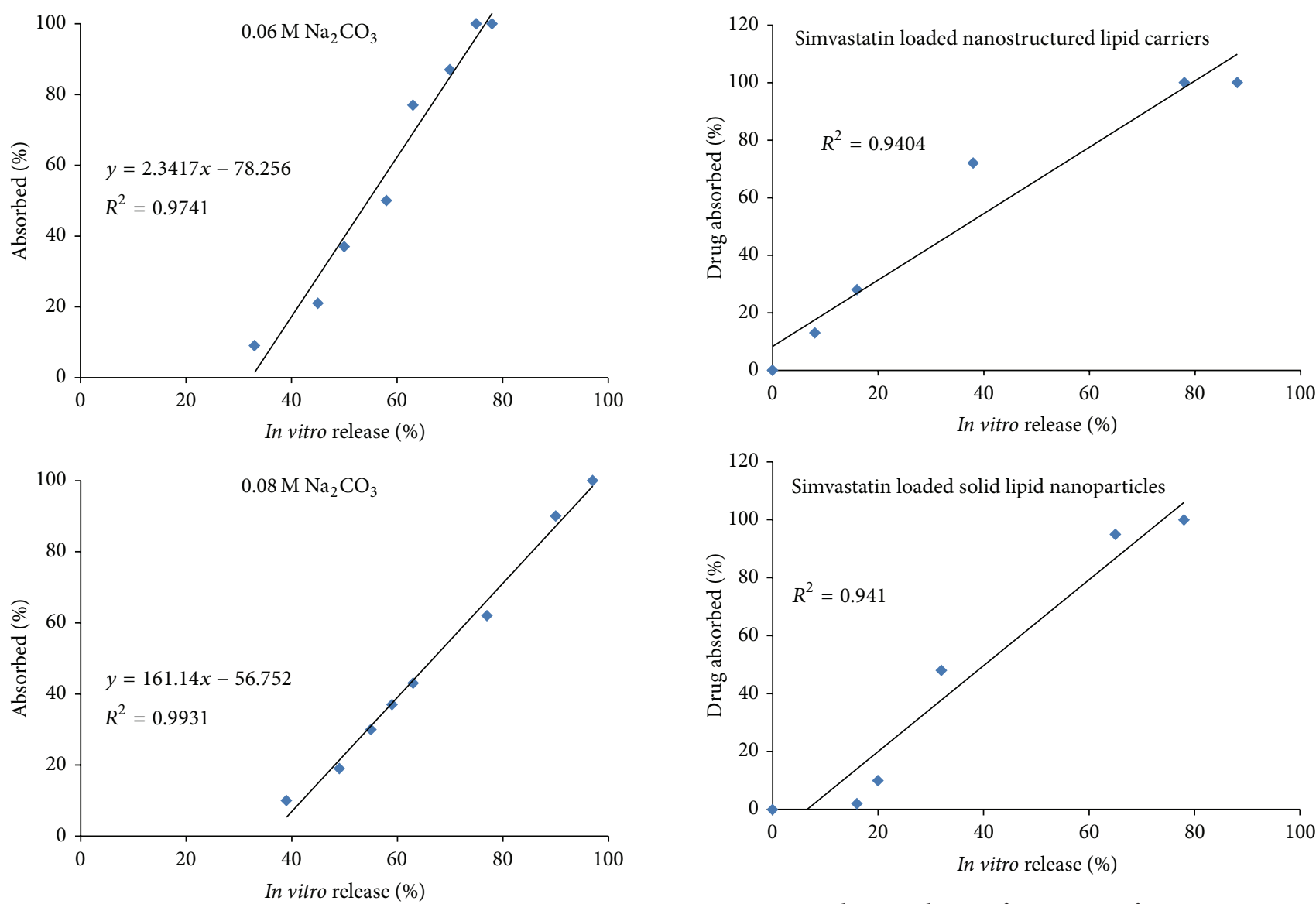

FIGURE 11: Level A IVIVC from Silybin meglumine hollow-type mesoporous silica nanoparticles using a combined USP II-dialysis bag set-up (redrawn from [33]).

correlation between in vitro release (dialysis bag) and percent drug absorbed (Wagner Nelson method) for Simvastatin nanostructured lipid carriers as well as nanoparticles $\left(R^{2}>\right.$ 0.94) (Figure 12) [34].

\section{Conclusions}

For novel dosage forms like nanoparticles where no regulatory or compendial standards exist, in vitro drug release assessment assumes greater significance in serving as an indicator of product quality and performance. A plethora of methods have been used, each with their advantages and drawbacks with respect to ease of set-up, sampling, and rapid buffer replacement. Ideally, an in vitro release method should simulate in vivo conditions, release mechanisms, and enable the establishment of an IVIVC. Future research should focus on developing relevant mathematical models to predict drug release behavior as well as release mechanisms applicable to a wide range of nano-sized dosage forms.

\section{Conflict of Interests}

The author declares that there is no conflict of interests regarding the publication of this paper.

FIGURE 12: Level A correlation of Simvastatin from two nano-sized dosage forms (redrawn from [34]).

\section{References}

[1] N. Al Khouri Fallouh, L. Roblot-Treupel, and H. Fessi, "Development of a new process for the manufacture of polyisobutylcyanoacrylate nanocapsules," International Journal of Pharmaceutics, vol. 28, no. 2-3, pp. 125-132, 1986.

[2] C. Losa, P. Calvo, E. Castro, J. L. Vila-Jato, and M. J. Alonso, "Improvement of ocular penetration of amikacin sulphate by association to poly(butylcyanoacrylate) nanoparticles," Journal of Pharmacy and Pharmacology, vol. 43, no. 8, pp. 548-552, 1991.

[3] C. Losa, L. Marchal-Heussler, F. Orallo, J. L. Vila Jato, and M. J. Alonso, "Design of new formulations for topical ocular administration: polymeric nanocapsules containing metipranolol," Pharmaceutical Research, vol. 10, no. 1, pp. 80-87, 1993.

[4] C. N. Cruz, K. M. Tyner, L. Velazquez et al., "CDER risk assessment exercise to evaluate potential risks from the use of nanomaterials in drug products," AAPS Journal, vol. 15, no. 3, pp. 623-628, 2013.

[5] G. Verreck, I. Chun, J. Rosenblatt et al., "Incorporation of drugs in an amorphous state into electrospun nanofibers composed of a water-insoluble, nonbiodegradable polymer," Journal of Controlled Release, vol. 92, no. 3, pp. 349-360, 2003.

[6] H. Heiati, R. Tawashi, R. R. Shivers, and N. C. Phillips, "Solid lipid nanoparticles as drug carriers I: incorporation and retention of the lipophilic prodrug 3 ' -azido-3' -deoxythymidine palmitate," International Journal of Pharmaceutics, vol. 146, no. 1, pp. 123-131, 1997. 
[7] M. Cetin, A. Atila, and Y. Kadioglu, "Formulation and in vitro characterization of Eudragit L100 and Eudragit L100-PLGA nanoparticles containing diclofenac sodium," AAPS PharmSciTech, vol. 11, pp. 1250-1256, 2010.

[8] A. Hanafy, H. Spahn-Langguth, G. Vergnault et al., "Pharmacokinetic evaluation of oral fenofibrate nanosuspensions and SLN in comparison to conventional suspensions of micronized drug," Advanced Drug Delivery Reviews, vol. 59, no. 6, pp. 419426, 2007.

[9] T. M. Allen, W. W. K. Cheng, J. I. Hare, and K. M. Laginha, "Pharmacokinetics and pharmacodynamics of lipidic nanoparticles in cancer," Anti-Cancer Agents in Medicinal Chemistry, vol. 6, no. 6, pp. 513-523, 2006.

[10] J. Kreuter, "Nanoparticle-based drug delivery systems," Journal of Controlled Release, vol. 16, no. 1-2, pp. 169-176, 1991.

[11] M. L. Etheridge, S. A. Campbell, A. G. Erdman, C. L. Haynes, S. M. Wolf, and J. McCullough, "The big picture on nanomedicine: the state of investigational and approved nanomedicine products," Nanomedicine: Nanotechnology, Biology, and Medicine, vol. 9, no. 1, pp. 1-14, 2013.

[12] S. Nie, "Understanding and overcoming major barriers in cancer nanomedicine," Nanomedicine, vol. 5, no. 4, pp. 523-528, 2010.

[13] W. H. de Jong and P. J. A. Borm, "Drug delivery and nanoparticles: applications and hazards," International Journal of Nanomedicine, vol. 3, no. 2, pp. 133-149, 2008.

[14] S. D'Souza, J. A. Faraj, R. Dorati, and P. P. DeLuca, "A short term quality control tool for biodegradable microspheres," AAPS PharmSciTech, vol. 15, no. 3, pp. 530-541, 2014.

[15] P. Buch, P. Holm, J. Q. Thomassen et al., "IVIVC for fenofibrate immediate release tablets using solubility and permeability as in vitro predictors for pharmacokinetics," Journal of Pharmaceutical Sciences, vol. 99, no. 10, pp. 4427-4436, 2010.

[16] S. D'Souza, J. A. Faraj, S. Giovagnoli, and P. P. DeLuca, "IVIVC from long acting olanzapine microspheres," International Journal of Biomaterials, vol. 2014, Article ID 407065, 11 pages, 2014.

[17] L. C. Amann, M. J. Gandal, R. Lin, Y. Liang, and S. J. Siegel, "In vitro-in vivo correlations of scalable PLGA-Risperidone implants for the treatment of schizophrenia," Pharmaceutical Research, vol. 27, no. 8, pp. 1730-1737, 2010.

[18] G. Zackrisson, G. Östling, B. Skagerberg, and T. Anfält, "ACcelerated Dissolution Rate Analysis (ACDRA) for controlled release drugs. Application to Roxiam," Journal of Pharmaceutical and Biomedical Analysis, vol. 13, no. 4-5, pp. 377-383, 1995.

[19] S. S. D'Souza, J. A. Faraj, and P. P. DeLuca, "A model-dependent approach to correlate accelerated with real-time release from biodegradable microspheres," AAPS PharmSciTech, vol. 6, article 70, no. 4, 2005.

[20] D. J. Burgess, A. S. Hussain, T. S. Ingallinera, and M. Chen, "Assuring quality and performance of sustained and controlled release parenterals: workshop report," AAPS PharmSci, vol. 4, article E7, 2002.

[21] S. S. D'Souza and P. P. DeLuca, "Methods to assess in vitro drug release from injectable polymeric particulate systems," Pharmaceutical Research, vol. 23, no. 3, pp. 460-474, 2006.

[22] M. Siewert, J. Dressman, C. K. Brown, and V. P. Shah, "FIP/AAPS guidelines to dissolution/in vitro release testing of novel/special dosage forms," AAPS PharmSciTech, vol. 4, article 7, 2003.

[23] C. K. Brown, H. D. Friedel, A. R. Barker et al., "FIP/AAPS joint workshop report: dissolution/in vitro release testing of novel/special dosage forms," AAPS PharmSciTech, vol. 12, no. 2, pp. 782-794, 2011.

[24] M. Martinez, M. Rathbone, D. Burgess, and M. Huynh, "In vitro and in vivo considerations associated with parenteral sustained release products: a review based upon information presented and points expressed at the 2007 Controlled Release Society Annual Meeting," Journal of Controlled Release, vol. 129, no. 2, pp. 79-87, 2008.

[25] M. N. Martinez, M. J. Rathbone, D. Burgess, and M. Huynh, "Breakout session summary from AAPS/CRS joint workshop on critical variables in the in vitro and in vivo performance of parenteral sustained release products," Journal of Controlled Release, vol. 142, no. 1, pp. 2-7, 2010.

[26] N. Chidambaram and D. J. Burgess, "A novel in vitro release method for submicron-sized dispersed systems," AAPS PharmSci, vol. 1, no. 3, pp. 32-40, 1999.

[27] M. M. A. Abdel-Mottaleb and A. Lamprecht, "Standardized in vitro drug release test for colloidal drug carriers using modified USP dissolution apparatus I," Drug Development and Industrial Pharmacy, vol. 37, no. 2, pp. 178-184, 2011.

[28] A. Maghsoudi, S. A. Shojaosadati, and E. Vasheghani Farahani, "5-Fluorouracil-loaded BSA nanoparticles: formulation optimization and in vitro release study," AAPS PharmSciTech, vol. 9, no. 4, pp. 1092-1096, 2008.

[29] U. Bhardwaj and D. J. Burgess, "A novel USP apparatus 4 based release testing method for dispersed systems," International Journal of Pharmaceutics, vol. 388, no. 1-2, pp. 287-294, 2010.

[30] L. Mora, K. Y. Chumbimuni-Torres, C. Clawson, L. Hernandez, L. Zhang, and J. Wang, "Real-time electrochemical monitoring of drug release from therapeutic nanoparticles," Journal of Controlled Release, vol. 140, no. 1, pp. 69-73, 2009.

[31] C. B. Michalowski, S. S. Guterres, and T. Dalla Costa, "Microdialysis for evaluating the entrapment and release of a lipophilic drug from nanoparticles," Journal of Pharmaceutical and Biomedical Analysis, vol. 35, no. 5, pp. 1093-1100, 2004.

[32] R. Kumar, R. C. Nagarwal, M. Dhanawat, and J. K. Pandit, "In-vitro and in-vivo study of indomethacin loaded gelatin nanoparticles," Journal of Biomedical Nanotechnology, vol. 7, no. 3, pp. 325-333, 2011.

[33] X. Cao, W. W. Deng, M. Fu et al., "In vitro release and in vitroin vivo correlation for silybin meglumine incorporated into Hollow-type mesoporous silica nanoparticles," International Journal of Nanomedicine, vol. 7, pp. 753-762, 2012.

[34] R. Tiwari and K. Pathak, "Nanostructured lipid carrier versus solid lipid nanoparticles of simvastatin: comparative analysis of characteristics, pharmacokinetics and tissue uptake," International Journal of Pharmaceutics, vol. 415, no. 1-2, pp. 232-243, 2011.

[35] X. Cao, W. Deng, M. Fu et al., "Seventy-two-hour release formulation of the poorly soluble drug silybin based on porous silica nanoparticles: in vitro release kinetics and in vitro/in vivo correlations in beagle dogs," European Journal of Pharmaceutical Sciences, vol. 48, no. 1-2, pp. 64-71, 2013.

[36] Y. Xu, Y. Wang, X. M. Li et al., "Study on the release of fenofibrate nanosuspension in vitro and its correlation with in situ intestinal and in vivo absorption kinetics in rats," Drug Development and Industrial Pharmacy, vol. 40, no. 7, pp. 972979, 2014.

[37] N. Borkar, D. Xia, R. Holm et al., "Investigating the correlation between in vivo absorption and in vitro release of fenofibrate from lipid matrix particles in biorelevant medium," European Journal of Pharmaceutical Sciences, vol. 51, pp. 204-210, 2014. 
[38] D. Heng, D. J. Cutler, H. K. Chan, J. Yun, and J. A. Raper, "What is a suitable dissolution method for drug nanoparticles?" Pharmaceutical Research, vol. 25, no. 7, pp. 1696-1701, 2008.

[39] A. C. Kilic, Y. Capan, I. Vural et al., "Preparation and characterization of PLGA nanospheres for the targeted delivery of NR2Bspecific antisense oligonucleotides to the NMDA receptors in the brain," Journal of Microencapsulation, vol. 22, no. 6, pp. 633641, 2005.

[40] Y. Zhang, H. Wang, C. Li et al., "A novel three-dimensional large-pore mesoporous carbon matrix as a potential nanovehicle for the fast release of the poorly water-soluble drug, celecoxib," Pharmaceutical Research, vol. 31, pp. 1059-1070, 2014.

[41] V. Sanna, A. M. Roggio, S. Siliani et al., "Development of novel cationic chitosan-and anionic alginate-coated poly(D,L-lactideco-glycolide) nanoparticles for controlled release and light protection of resveratrol," International Journal of Nanomedicine, vol. 7, pp. 5501-5516, 2012.

[42] F. Danhier, N. Lecouturier, B. Vroman et al., "Paclitaxel-loaded PEGylated PLGA-based nanoparticles: In vitro and in vivo evaluation," Journal of Controlled Release, vol. 133, no. 1, pp. 1117, 2009.

[43] Y. Li, Y. Pei, X. Zhang et al., "PEGylated PLGA nanoparticles as protein carriers: synthesis, preparation and biodistribution in rats," Journal of Controlled Release, vol. 71, no. 2, pp. 203-211, 2001.

[44] S. Prabha, W.-Z. Zhou, J. Panyam, and V. Labhasetwar, "Sizedependency of nanoparticle-mediated gene transfection: studies with fractionated nanoparticles," International Journal of Pharmaceutics, vol. 244, no. 1-2, pp. 105-115, 2002.

[45] S. J. Wallace, J. Li, R. L. Nation, and B. J. Boyd, "Drug release from nanomedicines: selection of appropriate encapsulation and release methodology," Drug Delivery and Translational Research, vol. 2, no. 4, pp. 284-292, 2012.

[46] D. Juenemann, E. Jantratid, C. Wagner, C. Reppas, M. Vertzoni, and J. B. Dressman, "Biorelevant in vitro dissolution testing of products containing micronized or nanosized fenofibrate with a view to predicting plasma profiles," European Journal of Pharmaceutics and Biopharmaceutics, vol. 77, no. 2, pp. 257-264, 2011.

[47] P. F. Yue, X. Y. Lu, Z. Z. Zhang et al., "The study on the entrapment efficiency and in vitro release of puerarin submicron emulsion," AAPS PharmSciTech, vol. 10, no. 2, pp. 376-383, 2009.

[48] M. Mahkam, F. Hosseinzadeh, and M. Galehassadi, "Preparation of ionic liquid functionalized silica nanoparticles for oral drug delivery," Journal of Biomaterials \& Nanobiotechnology, vol. 3, pp. 391-395, 2012.

[49] A. S. Ham, M. R. Cost, A. B. Sassi, C. S. Dezzutti, and L. C. Rohan, "Targeted delivery of PSC-RANTES for HIV-1 prevention using biodegradable nanoparticles," Pharmaceutical Research, vol. 26, no. 3, pp. 502-511, 2009.

[50] Y. I. Kim, L. Fluckiger, M. Hoffman, I. Lartaud-Idjouadiene, J. Atkinson, and P. Maincent, "The antihypertensive effect of orally administered nifedipine-loaded nanoparticles in spontaneously hypertensive rats," The British Journal of Pharmacology, vol. 120, no. 3, pp. 399-404, 1997.

[51] P. Liu, O. de Wulf, J. Laru et al., "Dissolution studies of poorly soluble drug nanosuspensions in non-sink conditions," AAPS PharmSciTech, vol. 14, no. 2, pp. 748-756, 2013.

[52] L. Sievens-Figueroa, N. Pandya, A. Bhakay et al., "Using USP I and USP IV for discriminating dissolution rates of nano- and microparticle-loaded pharmaceutical strip-films," AAPS PharmSciTech, vol. 13, no. 4, pp. 1473-1482, 2013.

[53] G. P. Yan, R. F. Zong, L. Li, T. Fu, F. Liu, and X. H. Yu, "Anticancer drug-loaded nanospheres based on biodegradable amphiphilic $\varepsilon$-caprolactone and carbonate copolymers," Pharmaceutical Research, vol. 27, no. 12, pp. 2743-2752, 2010.

[54] P. Calvo, J. L. Vila-Jato, and M. J. Alonso, "Comparative in vitro evaluation of several colloidal systems, nanoparticles, nanocapsules, and nanoemulsions, as ocular drug carriers," Journal of Pharmaceutical Sciences, vol. 85, no. 5, pp. 530-536, 1996.

[55] M. S. Muthu and S. Singh, "Poly (D, L-lactide) nanosuspensions of risperidone for parenteral delivery: formulation and in-vitro evaluation," Current Drug Delivery, vol. 6, no. 1, pp. 62-68, 2009.

[56] M. Y. Levy and S. Benita, "Drug release from submicronized o/w emulsion: a new in vitro kinetic evaluation model," International Journal of Pharmaceutics, vol. 66, no. 1-3, pp. 29-37, 1990.

[57] X. Xu, M. A. Khan, and D. J. Burgess, "A two-stage reverse dialysis in vitro dissolution testing method for passive targeted liposomes," International Journal of Pharmaceutics, vol. 426, no. 1-2, pp. 211-218, 2012.

[58] B. E. Kilfoyle, L. Sheihet, Z. Zhang, M. Laohoo, J. Kohn, and B. B. Michniak-Kohn, "Development of paclitaxel-TyroSpheres for topical skin treatment," Journal of Controlled Release, vol. 163, no. 1, pp. 18-24, 2012.

[59] S. Uprit, R. K. Sahu, A. Roy, and A. Pare, "Preparation and characterization of minoxidil loaded nanostructured lipid carrier gel for effective treatment of alopecia," Saudi Pharmaceutical Journal, vol. 21, pp. 379-385, 2013.

[60] B. Qiu, M. Ji, X. Song et al., "Co-delivery of docetaxel and endostatin by a biodegradable nanoparticle for the synergistic treatment of cervical cancer," Nanoscale Research Letters, vol. 7, article 666, 2012.

[61] S. S. D'Souza and P. P. DeLuca, "Development of a dialysis in vitro release method for biodegradable microspheres," AAPS PharmSciTech, vol. 6, no. 2, article 42, 2005.

[62] Y. Gao, J. Zuo, N. Bou-Chacra et al., "In vitro release kinetics of antituberculosis drugs from nanoparticles assessed using a modified dissolution apparatus," BioMed Research International, vol. 2013, Article ID 136590, 9 pages, 2013.

[63] M. Joshi, S. Pathak, S. Sharma, and V. Patravale, "Design and in vivo pharmacodynamic evaluation of nanostructured lipid carriers for parenteral delivery of artemether: nanoject," International Journal of Pharmaceutics, vol. 364, no. 1, pp. 119126, 2008.

[64] J. W. Kostanski and P. P. DeLuca, "A novel in vitro release technique for peptide-containing biodegradable microspheres," AAPS PharmSciTech, vol. 1, article 4, 2000.

[65] J. P. K. Tan, C. H. Goh, and K. C. Tam, "Comparative drug release studies of two cationic drugs from $\mathrm{pH}$-responsive nanogels," European Journal of Pharmaceutical Sciences, vol. 32, no. $4-5$, pp. 340-348, 2007.

[66] K. M. Rosenblatt, D. Douroumis, and H. Bunjes, "Drug release from differently structured monoolein/poloxamer nanodispersions studied with differential pulse polarography and ultrafiltration at low pressure," Journal of Pharmaceutical Sciences, vol. 96, no. 6, pp. 1564-1575, 2007.

[67] N. Charalampopoulos, K. Avgoustakis, and C. G. Kontoyannis, "Differential pulse polarography: a suitable technique for monitoring drug release from polymeric nanoparticle dispersions," Analytica Chimica Acta, vol. 491, no. 1, pp. 57-62, 2003. 
[68] P. Kayaert, B. Li, I. Jimidar, P. Rombaut, F. Ahssini, and G. Van den Mooter, "Solution calorimetry as an alternative approach for dissolution testing of nanosuspensions," European Journal of Pharmaceutics and Biopharmaceutics, vol. 76, no. 3, pp. 507-513, 2010.

[69] M. T. Crisp, C. J. Tucker, T. L. Rogers, R. O. Williams III, and K. P. Johnston, "Turbidimetric measurement and prediction of dissolution rates of poorly soluble drug nanocrystals," Journal of Controlled Release, vol. 117, no. 3, pp. 351-359, 2007.

[70] M. V. Chaubal and C. Popescu, "Conversion of nanosuspensions into dry powders by spray drying: a case study," Pharmaceutical Research, vol. 25, no. 10, pp. 2302-2308, 2008.

[71] A. Helle, S. Hirsjärvi, L. Peltonen, J. Hirvonen, S. K. Wiedmer, and T. Hyötyläinen, "Novel, dynamic on-line analytical separation system for dissolution of drugs from poly(lactic acid) nanoparticles," Journal of Pharmaceutical and Biomedical Analysis, vol. 51, no. 1, pp. 125-130, 2010.

[72] V. Domínguez-Villegas, B. Clares-Naveros, M. L. GarcíaLópez et al., "Development and characterization of two nanostructured systems for topical application of flavanones isolated from Eysenhardtia platycarpa," Colloids and Surfaces B: Biointerfaces, vol. 116, pp. 183-192, 2014.

[73] M. Barzegar-Jalali, K. Adibkia, H. Valizadeh et al., "Kinetic analysis of drug release from nanoparticles," Journal of Pharmacy and Pharmaceutical Sciences, vol. 11, no. 1, pp. 167-177, 2008.

[74] L. Zeng, L. An, and X. Wu, "Modeling drug-carrier interaction in the drug release from nanocarriers," Journal of Drug Delivery, vol. 2011, Article ID 370308, 15 pages, 2011.

[75] L. Zeng and X. Wu, "Modeling the sustained release of lipophilic drugs from liposomes," Applied Physics Letters, vol. 97, no. 7, Article ID 073701, 2010.

[76] FDA Guidance for Industry, Extended Release Oral Dosage Forms: Development, Evaluation and Application of In Vitro/In Vivo Correlations, 1997.

[77] J. G. Wagner and E. Nelson, "Per cent absorbed time plots derived from blood level and/or urinary excretion data," Journal of Pharmaceutical Sciences, vol. 52, pp. 610-611, 1963. 

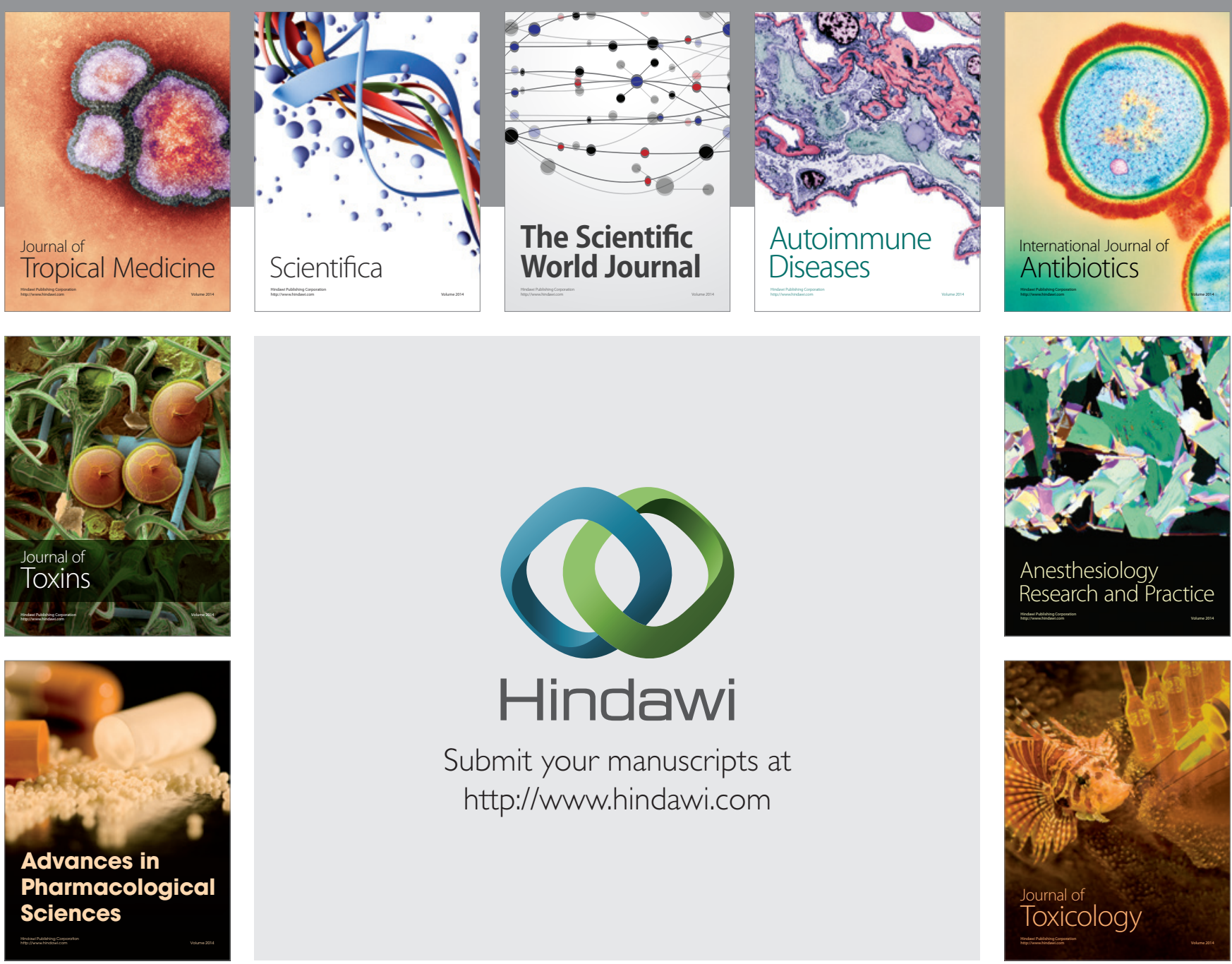

\section{Hindawi}

Submit your manuscripts at

http://www.hindawi.com
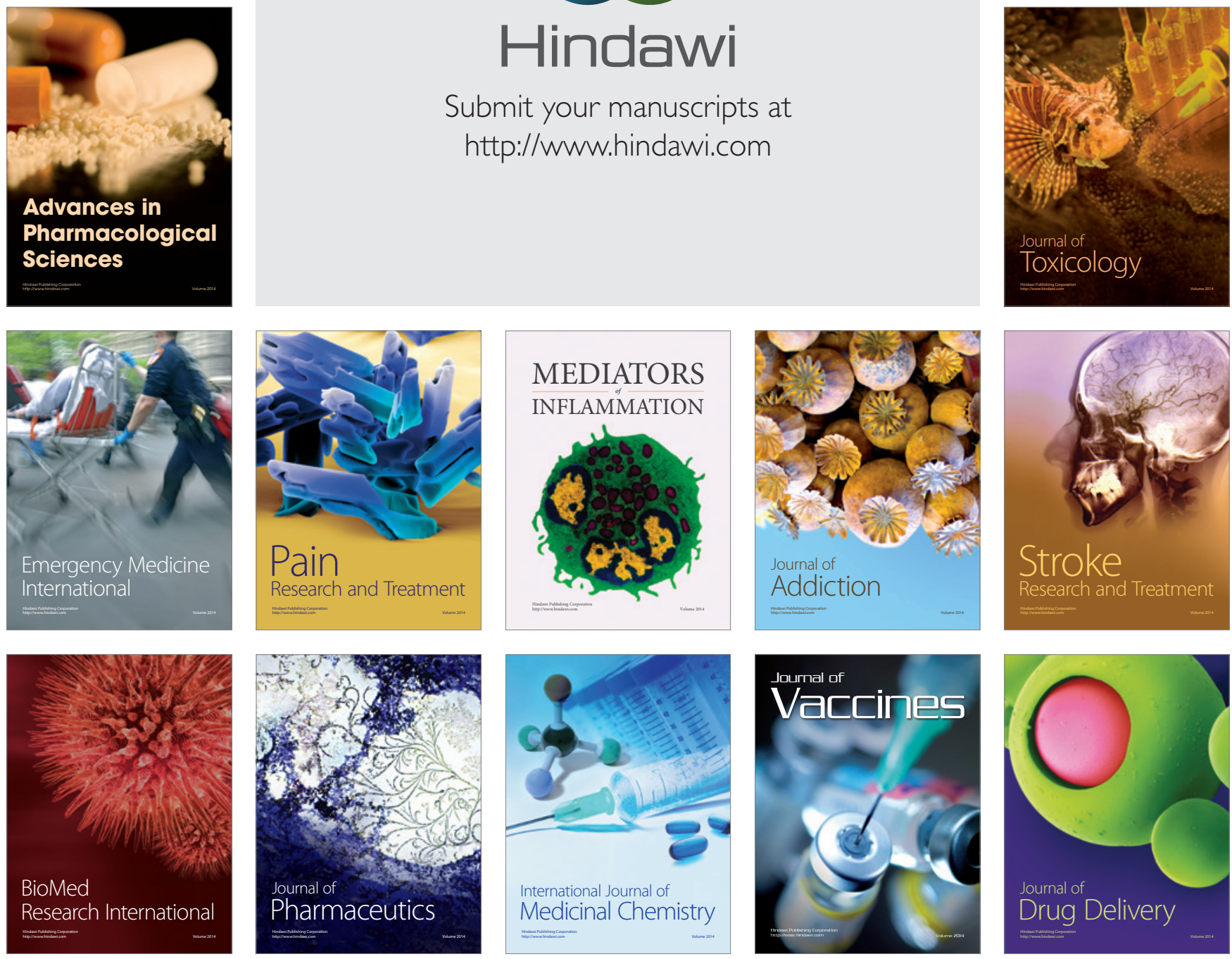